erhalten, Blättchen aus Ligroin, Sdp. $207^{\circ}$ unter $11 \mathrm{~mm}$, Smp. 130, dessen Salze ebenfalls mit Ferrichlorid die blaue Farbreaktion, aber weniger intensiv, zeigen. Aber die Methylierung gelingt nur sehr schwer; trotz der Einwirkung eines 10-fachen Überschusses von Dimethylsulfat und 30-proz. Natronlauge während 48 Stunden wurde im wesentlichen nur die Dimethylaminogruppe in die quaternäre Base verwandelt, ohne dass die Phenylaminogruppe reagiert hätte. Die beste Probe von Jodmethylat aus zahlreichen Versuchen ergab weisse Blättchen vom Smp. $198-199^{\circ}$.

$$
\begin{array}{ll}
0,2773 \text { gr Subst. gaben 0,1786 gr AgJ } \\
\mathrm{C}_{15} \mathrm{H}_{19} \mathrm{~N}_{2} \mathrm{~J} \text { (quaternär-sekundär) } & \text { Ber. J } 35,48 \% \\
\mathrm{C}_{16} \mathrm{H}_{21} \mathrm{~N}_{2} \mathrm{~J} \text { (quaternär-tertiär) } & \text { Ber. " } 34,47 \% \\
& \text { Gef. ", 34,81\% }
\end{array}
$$

Das Jodmethylat, mit Natronkalk im Vakuum destilliert, gab ein Basengemisch vom Smp. 123-1240, aus dem nur am Rand einige Nädelchen Smp. $57^{\circ}$ herauswuchsen, viel zu wenig, um eine genauere Untersuchung zu gestatten.

Ubber die Fortsetzung dieser sowie über synthetische Versuche in anderer Richtung soll gelegentlich später berichtet werden.

Basel, Anstalt für Anorganische Chemje, September 1921.

\title{
Polysaccharide XIV. Zur Kenntnis der Amylosen von
}

\section{P. Karrer und Elisabeth Bürklin.}

\section{(1. I. 22.)}

Aus dem Abbau der $\beta$-Hexamylose mit Acetylbromid und aus der Zusammensetzung der Natriumhydroxyd- $\beta$-hexamylose hat der eine von uns früher den Schluss gezogen, dass die $\beta$-Hexamylose eine polymere Form eines Disaccharid-anhydrids darstellen muss und nicht diejenige eines Trisaccharid-anhydrids. Um darüber weitere Klarheit zu gewinnen, haben wir nun die durch Acetylierung der $\beta$-Hexamylose hergestellte sogenannte Triamylose in den Kreis unserer Untersuchung einbezogen. Dieselbe ist in Wasser von $20^{\circ}$ recht schwer löslich, was der Grund gewesen sein mag, warum Pringsheim sich auf die Molekulargewichtsbestimmung der acetylierten Triamylose beschränkt und eine solche mit der freien Triamylose nicht vorgenommen hatte. 
Die „Triamylose" stellten wir genau nach der Vorschrift von H. Pringsheim her.

Die aus ihrer Acetylverbindung isolierte und öfter umkrystallisierte Triamylose wurde hierauf im Verhalten gegen Acetylbromid geprüft. Wir fanden, dass aus ihr bei $0^{0}$ bis $+5^{0}$ mit Acetylbromid und sehr wenig Eisessig genau dieselbe Menge Acetobrom-maltose und Heptacetylmaltose gebildet wird, wie aus der entsprechenden Menge Maltose selbst; die Triamylose verhält sich in dieser Beziehung wie $\alpha$-Dianylose, $\beta$-Hexamylose oder Zulkowsky'sche Stärke. Aus $1 \mathrm{gr}$ Triamylose erhielten wir $0,533 \mathrm{gr}$ reine Heptacetyl-maltose vom Smp. $178^{\circ}$, während nach den Erfahrungen an der Maltose (80\% Ausbeute an Acetobrom-maltose, davon maximal $30 \%$ des Gewichtes Heptacetyl-maltose) aus 1 gr „Triamylose" maximal 0,505 gr Heptacetyl-maltose erwartet werden durften, sofern keine Triamylose, sondern eine Verbindung $\left(\mathrm{C}_{\mathbf{1 2}} \mathrm{H}_{\mathbf{2 0}} \mathrm{O}_{10}\right)_{\mathbf{x}}$ vorlag. Unsere Acetylbromidspaltung verlief also besonders glatt und wir konnten in diesem Fall selbst mit der Osazonprobe keine Glucose nach der Acetolyse nachweisen.

Dieser Versuch lehrt, dass die Triamylose kein Trisaccharid-anhydrid, sondern ein Maltose-anhydrid oder eine polymere Form eines solchen sein muss. Wir wollen das Ergebnis der weiteren Untersuchung gleich vorweg nehmen: es hat sich gezeigt, dass die Triamylose identisch ist mit der $\beta$-Hexamylose und dass somit bei der Acetylierung der $\beta$-Hexamylose mit Essigsäure-anhydrid und wenig Zinkchlorid eine Depolymerisation der $\beta$-Hexamylose nicht stattfindet. In allen Eigenschaften stimmt die Triamylose mit der $\beta$-Hexamylose überein, wie aus folgenden Angaben hervorgeht:

1. Die Natriumhydroxydverbindung der Triamylose, die wir in der an ähnlichen Körpern schon öfter beschriebenen Weise herstellten, hatte folgenden Natriumgehalt:

$$
\left(\mathrm{C}_{12} \mathrm{H}_{20} \mathrm{O}_{10} \cdot \mathrm{NaOH}\right)_{x} \quad \text { Ber. Na } 6,31 \% \text { Gef. Na 6,72\% }
$$

2. Der Pankreassaft, mit dem wir $\alpha$-Diamylose und $\alpha$-Tetramylose zu reduzierendem Zucker abbauen konnten, der aber die $\beta$-Hexamylose nicht angriff, war auch auf die Triamylose ohne Einwirkung.

3. Der Krystallwassergehalt der aus Wasser krystallisierten $\beta$-Hexaamylose war $14,26 \%$, dies entspricht $9 \mathrm{Mol}$.Krystallwasser $\left(\mathrm{für}\left(\mathrm{C}_{\mathbf{1 2}} \mathrm{H}_{\mathbf{2 0}} \mathrm{O}_{\mathbf{1 0}}\right)_{\mathbf{3}}\right.$ - $9 \mathrm{H}_{2} \mathrm{O}$ berechnen sich $14,28 \% \mathrm{H}_{2} \mathrm{O}$ ), wie schon $\mathrm{H}$. Pringsheim gefunden hatte. In der in gleicher Weise hergestellten krystallisierten Triamylose fanden wir $14,17 \%$ Krystallwasser, somit genau gleich viel.

4. Die Löslichkeit der $\beta$-Hexamylose in Wasser von $18^{\circ}$ bestimmten wir zu 2,1\%; die Löslichkeit der Triamylose war unter denselben Bedingungen $2,2 \%$. 
5. Die spez. Drehung der $\beta$-Hexamylose ist in reinem Zustand $+157^{\circ}$ bis $158^{\circ}$. Diejenige der Triamylose fanden wir in einem Präparat $+156,5^{\circ}$, in einem anderen $+158,4^{\circ}$, in einem dritten $+158,6^{\circ}$.

6. Molekulargewichtsbestimmungen der Triamylose lassen sich leider in Wasser nicht genau ausführen; die Löslichkeit ist zu gering und die Ausschläge sind daher zu klein.

7. Die krystallographische Untersuchung der $\beta$-Hexamylose und der Triamylose, die in freundlicher Weise durch Herrn Kollegen Prof. P. Niggli und Herrn E. Widmer ausgeführt wurde, ergab folgendes Resultat:

„Es krystallisieren beide Körper monoklin und zeigen folgende Formen: a $\{100\}$ (nach welcher der Habitus beider Körper ein tafeliger ist); $\mathrm{m}\{110\}$ und $\mathrm{c}\{001\}$, ferner $\mu\{1 \overline{1} 0\}, q\{011\}$, o $\{111\}, \varrho\{10 \overline{1}\}$. Die Flächen der Zone [001] sind parallel c gestreift.

An den bis jetzt gemessenen Krystallen trat mit der Fläche

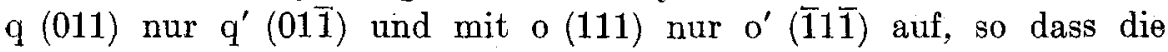
monoklin hemimorphe Symmetrie vorzuliegen scheint.

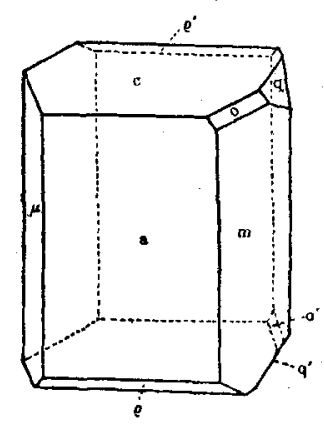

Flächenwinkel:

$\begin{array}{cr}\text { Triamylose } & \beta \text {-Hexamylose } \\ \mathrm{a}: \mathrm{c} \text { Mittel } 69^{\circ} 05^{\prime} & \text { Mittel } 69^{\circ} 06^{\prime} \\ \mathrm{m}: \mathrm{m}^{\prime} \quad 5^{\circ} 28^{\prime} & 55^{\circ} 32^{r}\end{array}$

Spaltbarkeit:

Triamylose

B-Hexamylose

$\operatorname{nach}\{010\}\{001\}\{100\}$

$\operatorname{nach}\{010\}\{001\}\{100\}$

Optisches Verhalten:

Triamylose

ק-Hexamylose

Gerade Auslöschung auf

$\{100\}$ Gerade Auslöschung auf

$\{100\}$

Auf Spaltblättchen parallel $\{010\}$ :

Auf Spaltblättchen

$\{010\}$ :

Die opt. Achsenebene bildet mit c Die opt. Achsenebene bildet mit c einen Winkel von ca. $22^{\circ} \mathrm{im}$ stumpfen Winkel $\beta$. einen Winkel von $22^{\circ}$ im stumpfen Winkel $\beta$.

Auf $\{010\}$ tritt die spitze, negative Bisektrix $\left(\mathbf{n}_{\alpha}\right)$ aus. Der seheinbare Winkel der optischen Achsen beträgt schätzungsweise $50-60^{\circ}$. 
Aus diesen vergleichenden Daten geht klar hervor, dass $\beta$-Hexaamylose und Triamylose identisch sind, und dass somit die Acetylierung $\operatorname{der} \beta$-Hexamylose mit Essigsäure-anhydrid-Zinkchlorid ohne Depolymerisation verläuft. Die Unterschiede, die man seinerzeit an den Eigenschaften der $\beta$-Hexamylose und Triamylose beobachtet hatte, sind nicht so gross, dass sie nicht auf geringe Verunreinigung oder Zufälligkeiten zurückgeführt werden könnten; sie beschränken sich auf den Krystallwassergehalt, wo Pringsheim 12,4 und $13,4 \%$ statt wie wir $14,11 \%$ gefunden hatte ( $\beta$-Hexamylose $14,2 \%$ ), und auf die Werte der spezifischen Drehung, wo Pringsheim ca. $152^{\circ}$, wir ca. $157^{\circ}-158^{\circ}$ fanden $(\beta$-Hexamylose 157-158 $)$. Dagegen hat schon Pringsheim gezeigt, dass die Jod- wie die Bromadditionsprodukte von Triamylose und $\beta$-Hexamylose genau dieselbe prozentuale Zusammensetzung haben.

Es bleibt noch die Frage, warum die Molekulargewichtsbestimmung der sogenannten acetylierten Triamylose zu kleine Werte ergeben hatte - auf ihr beruht ja die Auffassung der Verbindung als Triamylose. Wir haben auch an den Acetylverbindungen anderer Anhydrozucker die Beobachtung gemacht, dass deren Molekulargewichtsbestimmung unter Umständen ungenaue oder unrichtige Werte ergibt und werden in einer spätern Mitteilung solche Fälle beschreiben. Es scheint uns daher angebracht, Molekulargewichtsbestimmungen acylierter Anhydrozucker nur mit Vorsicht zu verwerten; diese Warnung ist um so notwendiger, als neuerdings dieses Verfahren zur Ermittlung der Molekelgrösse der Anhydrozucker verschiedentlich benutzt worden ist. Im oben beschriebenen Fall hat es jedenfalls versagt.

Unsere Befunde scheinen uns in verschiedener Hinsicht von allgemeinerem Interesse zu sein.

Erstens verschwindet mit der Triamylose aus der Stärkechemie das letzte Polysaccharid, das aus mehr wie zwei Glucoseresten sich aufbaut. Wir stehen jetzt der Tatsache gegenüber, dass aus Stärke auf allen bisher betretenen Wegen nur Maltose bezw. Maltose-anhydrid und dessen polymere Formen isoliert werden konnten, wie dies die neue Auffassung vom Stärkeaufbau verlangt. Wahrscheinlich ist die $\beta$-Hexamylose polymerisationsisomer mit den $\alpha$-Amylosen. Bemerkenswert bleibt, dass solche Isomere, wie sie die $\alpha$-Amylosen und $\beta$-Hexamylose sind, schon durch Erhitzen in Lösungsmitteln ineinander umgewandelt werden.

Zweitens zeigt der Fall der $\beta$-Hexamylose, dass es polymere Anhydrozucker gibt, die sich beim Acetylieren mit Essigsäure-anhydrid und wenig Zinkchlorid nicht depolymerisieren. Dies ist darum von Bedeutung, weil man nach dieser Methode auch Cellulose, Stärke etc. 
häufig acetyliert hat, meistens allerdings bei Anwesenheit von etwas. grösseren Mengen Zinkchlorid, und geneigt ist, anzunehmen, dass hierbei ein mehr oder weniger tiefer Abbau eintrete. Ost hat aber schon darauf hingewiesen, dass von den zur Acetylierung zugesetzten Katalysatoren Zinkchlorid die Cellulose am wenigsten zerstört, und der Versuch scheint nicht aussichtlos, die Acetylierung so zu leiten, dass ein Abbau überhaupt nicht Platz greift. Es öffnet sich hier ein grösserer Fragenkomplex, auf den wir in einer spätern Abhandlung zurückzukommeń beabsichtigen.

Endlich zeigt der Fall $\beta$-Hexamylose-Triamylose, wie leistungsfähig unsere Acetylbromidspaltung und Untersuchungsmethode mittelst. den Alkalihydroxyd-additionsverbindungen zur Konstitutionsermittlung polymerer Anhydrozucker sind, denn sie waren es ja, die uns zuerst anzeigten, dass die $\beta$-Hexamylose keine polymere Triamylose, sondern ein polymeres Maltose-anhydrid sein muss.

\section{Experimenteller Teil.}

Da es sich im folgenden um einen Vergleich zwischen der $\beta$-Hexaámylose und der Triamylose handelt, so verwenden wir für letztere noch die Bezeichnung ,Triamylose"; wir wissen nun, dass diese eine über die Acetylverbindung regenerierte $\beta$-Hexamylose ist.

\section{Überführung der „Triamylose" in Heptacetyl-maltose.}

1 gr ,Triamylose“ wurde mit 10 gr Acetylbromid und 6 Tropfen Eisessig in einem mit eingeschliffenem Kühler und Calciumchloridrohr versehenen Kolben 48 Stunden bei $0^{0}$ stehen gelassen. Hierbei trat Lösung ein. Dann wurde auf Eis gegossen, die rohe Acetobrommaltose fiel aus. Sie wurde abgenutscht, mit Wasser gewaschen, in Äther gelöst. und die Ätherlösung nach der Filtration eine halbe Stunde mit Silbercarbonat geschüttelt. Hierauf wurde der Niederschlag, aus Silberverbindungen und Heptacetyl-maltose bestehend, abgenutscht, mit etwas. Äther nachgewaschen und nachher zweimal mit Chloroform ausgekocht. Der Chloroformauszug hinterliess beim Eindampfen 0,533 gr reine Heptacetyl-maltose vom Smp. $178^{\circ}$.

In dem Äther, der vom Niederschlag der Heptacetyl-maltose und den Silberverbindungen abgenutscht worden war, suchten wir nach Glucose. Wir verdampften den Äther, liessen den Rückstand zur Verseifung der Acetylverbindungen mit n. Natronlauge stehen und erhitzten hierauf die schwach essigsaure Lösung mit Phenylhydrazin. 


\section{$-186$}

Schwer lösliches Glucosazon beobachteten wir dabei nicht. Es können daher bei der Acetylbromidspaltung der Triamylose höchstens sehr kleine Mengen Traubenzucker entstanden sein.

\section{Natriumhydroxyd-additionsverbindung der ,Triamylose“.}

$0,5 \mathrm{gr}$ Triamylose wurden in $3 \mathrm{~cm}^{3}$ reiner, 10-proz. Natronlauge gelöst, die Lösung unter Rühren in ca. $100 \mathrm{~cm}^{3}$ absoluten Alkohol gegossen, das ausgefallene weisse Pulver abgenutscht, dreimal mit absolutem Alkohol nachgewaschen, hierauf in möglichst wenig $\left(\mathrm{ca.} 2 \mathrm{~cm}^{3}\right)$ Wasser gelöst und nochmals in gleicher Weise in absolutem Alkohol gefällt. Nach dem Trocknen bei $100^{\circ}$ im Vakuum über Phosphorpentoxyd ergab die Analyse folgenden Natriumgehalt:

0,0498 gr Subst. erforderten zur Neutralisation $1,4 \mathrm{~cm}^{3}$ Schwefelsäure ( $1 \mathrm{~cm}^{3}$ enthält $0,005095 \mathrm{gr} \mathrm{H}_{2} \mathrm{SO}_{4}$ )

$$
\left(\mathrm{C}_{13} \mathrm{H}_{20} \mathrm{O}_{10} \cdot \mathrm{NaOH}\right)_{3} \quad \text { Ber. Na } 6.31 \% \text { Gef. Na } 6,72 \%
$$

Krystallwasserbestimmung in der $\beta$-Hexamylose und ,Triamylose“.

Beide Präparate waren nebeneinander in derselben Weise aus Wasser krystallisiert, und nachher gleich lang an der Luft getrocknet.

0,1508 gr $\beta$-Hexamylose verloren im Trockenschrank bei $105-110^{\circ}$

$$
0,0215 \text { gr } \mathrm{H}_{2} \mathrm{O}=14,26 \% \mathrm{H}_{2} \mathrm{O}
$$

0,1403 gr Triamylose verloren im Trockenschrank bei $105-110^{0}$

$$
0,0199 \text { gr } \mathrm{H}_{2} \mathrm{O}=14,17 \% \mathrm{H}_{2} \mathrm{O}
$$

Ber. für $\left(\mathrm{C}_{12} \mathrm{H}_{20} \mathrm{O}_{10}\right)_{3} \cdot 9 \mathrm{H}_{2} \mathrm{O} \quad \mathrm{H}_{2} \mathrm{O} \quad 14,28 \%$

Bestimmung der Wasserlöslichkeit von $\beta$-Hexamylose und ,Triamylose“.

0,5166 gr getrocknete $\beta$-Hexamylose wurden bei $18^{\circ}$ drei Stunden mit $20 \mathrm{~cm}^{3}$ Wasser

auf der Maschine geschüttelt. Ungelöster, getrockneter Rückstand 0,1021 gr

Löslichkeit der $\beta$-Hexamylose $2,07 \%$

$0,5166 \mathrm{gr}$ "Triamylose" wurden mit $20 \mathrm{~cm}^{3}$ Wasser bei $18^{\circ}$ drei Stunden auf der Maschine geschüttelt. Ungelöster, getrockneter Rückstand $0,0708 \mathrm{gr}$

Löslichkeit im Wasser 2,22\%

Polarisation der Triamylose.

1. Präparat:

0,0900 gr Subst. Gesamtgewicht der wässerigen Lösung 10,314 gr, d=1,003

$$
\begin{aligned}
& \text { Rohrlänge } 0,5 \mathrm{dm} . \quad a_{\mathrm{D}}^{19}=+0,685^{0} \\
& {[a]_{\mathrm{D}}^{19}=\frac{+0,685 \times 10,314}{0,5 \times 1,003 \times 0,0900}=+156,5^{0}}
\end{aligned}
$$


2. Präparat:

0,1059 gr Subst. Gesamtgewicht der wässerigen Lösung 11,074 gr, d=1,003

$$
\begin{aligned}
& \text { Rohrlänge } 1 \mathrm{dm} . \quad a_{D}^{18}=+1,52^{\circ} \\
& {[\alpha]_{D}^{18}=\frac{+1,52 \times 11,074}{1 \times 1,003 \times 0,1059}=+158,4^{\circ}}
\end{aligned}
$$

3. Präparat:

$$
[a]_{D}^{16}=+158,6^{0}
$$

Zürich, Chemisches Laboratorium der Universität.

\section{Polysaccharide XV'). \\ Die Konstitution der Diamylose und des der Cellulose zu Grunde liegenden Anhydrozuckers (Cellosan) \\ von \\ P. Karrer und Alex. P. Smirnoff.}

(2. II. 22.)

Kürzlich haben wir eine neue Methode zur Konstitutionsbestimmung der Anhydrozucker beschriebèn ${ }^{2}$ ). Sie besteht darin, dass die Sanerstoffbrücke des Anhydrozuckers durch Einwirkung von Phosphorpentabromid geöffnet wird, wobei an die Kohlenstoffatome, die vorher Ausgangs- und Endpunkte der Sauerstoffbrücke gewesen waren, an Stelle des Sauerstoffs, Bromatome treten. So gab Triacetyl-lävoglucosan (I) mit Phosphorpentabromid Aceto-1,6-dibromglucose (II)

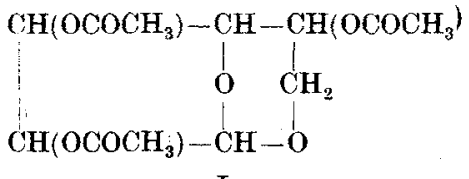

I.

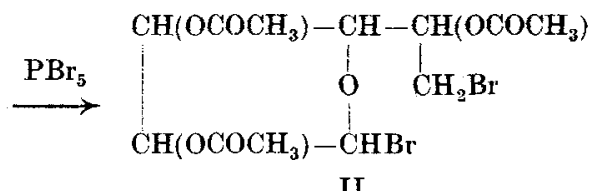

II.

1) XIV. Abhandlung Helv. 5, 181 (1922).

2) Helv. 5, 124 (1922). 